Proc. Indian Acad. Sci. (Math. Sci.) Vol. 115, No. 4, November 2003, pp. 383-389.

Printed in India

\title{
An algebra of absolutely continuous functions and its multipliers
}

\author{
SAVITA BHATNAGAR \\ Department of Mathematics, Panjab University, Chandigarh 160 014, India \\ E-mail: bhsavita@pu.ac.in
}

MS received 28 February 2005; revised 9 September 2005

\begin{abstract}
The aim of this paper is to study the algebra $A C_{p}$ of absolutely continuous functions $f$ on $[0,1]$ satisfying $f(0)=0, f^{\prime} \in L^{p}[0,1]$ and the multipliers of $A C_{p}$.
\end{abstract}

Keywords. Absolutely continuous function; Banach algebra; multiplier.

\section{Introduction}

Let $I=[0,1]$ be the compact topological semigroup with max multiplication and usual topology. $C(I), L^{p}(I), 1 \leq p \leq \infty$ are the associated Banach algebras. Larsen [5] obtained multipliers for the Banach algebra $L^{1}(I)$. Baker, Pym and Vasudeva [1] obtain characterizations of multipliers from $L^{p}(I)$ to $L^{r}(I), 1 \leq r, p \leq \infty$. Bhatnagar and Vasudeva [2] characterize $\operatorname{Hom}_{C(I)}\left(L^{r}(I), L^{p}(I)\right)$ and their pre-duals for $1 \leq r \leq p$. Bhatnagar [3] studied $\operatorname{Hom}_{C(I)}\left(L^{r}(I), L^{p}(I)\right)$ and their pre-duals for $r>p$. The study of pre-duals of the multipliers in [2] and [3] involved a deep understanding of the interpolation theory and lengthy calculations. The multipliers can be obtained more attractively by abstract arguments. It turns out that results obtained via abstract arguments compare with those obtained in [2] and [3]. Our results also include the results obtained by Larsen [5]. $L^{p}(I)$ is replaced by the Gelfand transform algebra $A C_{p}, p \geq 1$ of absolutely continuous functions $f$ on $[0,1]$ with $f(0)=0$ and $f^{\prime} \in L^{p}(I)$. With the norm defined as $\|f\|\|=\| f^{\prime} \|_{p}, f \in A C_{p}, A C_{p}$ constitute subalgebras of $C(I)$ and have an approximate identity. The purpose of this note is to study the multipliers from $A C_{r}$ to $A C_{p}, 1 \leq r, p \leq \infty$. For $r=p$, a complete description of multipliers is obtained. For $r<p$, the multiplier algebra consists of $\{0\}$ alone. In case of $r>p$ we provide a set of necessary and another set of sufficient conditions for a function in $C(0,1]$ to be a multiplier. An example of a function which satisfies the necessary conditions but does not satisfy the sufficient conditions and fails to be a multiplier is also provided.

\section{The Banach algebra $A C_{p}$}

Let $I=[0,1]$ with the usual interval topology be the compact metric space and $C(I)$ be the set of all continuous complex valued functions on $I$. For $1 \leq p \leq \infty$, let

$$
\begin{aligned}
A C_{p}= & \{f \in C(I): f \text { is absolutely continuous on } I, f(0)=0 \\
& \text { and } \left.f^{\prime} \in L^{p}(I)\right\} .
\end{aligned}
$$


Define $\||f|\|=\left\|f^{\prime}\right\|_{p}, f \in A C_{p}$. For $1 \leq p \leq \infty$, let $p^{\prime}$ denote the conjugate index to $p$, that is, $\frac{1}{p}+\frac{1}{p^{\prime}}=1$.

The following inequality will be frequently used in the sequel. For $g \in A C_{p}, 1<p<\infty$,

$$
\begin{aligned}
& |g(t)|=\left|\int_{0}^{t} g^{\prime}(s) \mathrm{d} s\right| \leq \int_{0}^{t}\left|g^{\prime}(s)\right| \mathrm{d} s \leq\left(\int_{0}^{t}\left|g^{\prime}(s)\right|^{p} \mathrm{~d} s\right)^{1 / p} t^{1 / p^{\prime}} \leq\left\|g^{\prime}\right\|_{p}, \\
& \text { for } g \in A C_{1},|g(t)| \leq\left\|g^{\prime}\right\|_{1}, \text { and for } g \in A C_{\infty},|g(t)| \leq\left\|g^{\prime}\right\|_{\infty} . t,
\end{aligned}
$$

using Holder's inequality.

Lemma 1 . Let $f$ be continuous on $[0,1]$, absolutely continuous on $[\alpha, 1]$ for any $\alpha$ with $0<\alpha \leq 1$ with $f^{\prime} \in L^{1}[0,1]$. Then $f$ is absolutely continuous on $[0,1]$.

The proof is elementary.

Theorem 2. $A C_{p}$ is a Banach subalgebra of $C(I)$. It has an approximate identity for $1 \leq$ $p<\infty . A C_{\infty}$ has no approximate identity. The maximal ideal space $\triangle\left(A C_{p}\right)$ of $A C_{p}, 1 \leq$ $p \leq \infty$ is homeomorphic to $(0,1]$. Moreover, $A C_{r} \subseteq A C_{p}$ for $r>p$.

Proof. Clearly $A C_{p}$ is a Banach space. For $f, g \in A C_{p}$,

$$
(f g)^{\prime}=f^{\prime} g+f g^{\prime} \in L^{p}(I)
$$

as $f^{\prime}, g^{\prime} \in L^{p}(I)$ and $f, g$ are bounded. Moreover,

$$
\|f g\|=\left\|(f g)^{\prime}\right\|_{p} \leq\|g\|_{\infty}\left\|f^{\prime}\right\|_{p}+\|f\|_{\infty}\left\|g^{\prime}\right\|_{p}
$$

Since $g \in A C_{p},|g(t)| \leq\left\|g^{\prime}\right\|_{p}=\|\| g\|\|$, using (1), and so $\|g\|_{\infty} \leq\|\| g\|\|$.

Similarly $\|f\|_{\infty} \leq\|\| f\|\|$. Consequently, $\left\|\left|f g\|\mid \leq 2\|\|f\|\|\|\|g\|\right.\right.$. Thus $A C_{p}$ is a Banach algebra.

Define $e_{\alpha}(t)=\min \{t / \alpha, 1\}, t \in I$. Then $e_{\alpha}^{\prime}(t)=\frac{1}{\alpha} \chi_{[0, \alpha)}(t)$. We show that $\left\{e_{\alpha}\right\}$ forms an approximate identity for $A C_{p}, 1 \leq p<\infty$ as $\alpha \rightarrow 0+$.

Now

$$
\begin{aligned}
\left\|e_{\alpha} g-g\right\| \| & =\left\|\left(e_{\alpha}-1\right) g^{\prime}+e_{\alpha}^{\prime} g\right\|_{p} \\
& \leq\left\|\left(e_{\alpha}-1\right) g^{\prime}\right\|_{p}+\left\|e_{\alpha}^{\prime} g\right\|_{p} . \\
\left\|e_{\alpha}^{\prime} g\right\|_{p} & =\left(\int_{0}^{\alpha}\left|\frac{1}{\alpha} g(t)\right|^{p} \mathrm{~d} t\right)^{1 / p} \\
& \leq \frac{1}{\alpha}\left(\int_{0}^{\alpha}\left(\int_{0}^{t}\left|g^{\prime}(s)\right|^{p} \mathrm{~d} s\right) t^{p / p^{\prime}} \mathrm{d} t\right)^{1 / p}, \quad \text { using (1). }
\end{aligned}
$$

So

$$
\begin{aligned}
\left\|e_{\alpha}^{\prime} g\right\|_{p} & \leq \frac{1}{\alpha}\left\|g^{\prime} \chi_{[0, \alpha]}\right\|_{p}\left(\int_{0}^{\alpha} t^{p / p^{\prime}} \mathrm{d} t\right)^{1 / p} \\
& =\frac{1}{\alpha}\left\|g^{\prime} \chi_{[0, \alpha]}\right\|_{p}\left(\frac{\alpha^{p}}{p}\right)^{1 / p} \\
& =p^{-1 / p}\left\|g^{\prime} \chi_{[0, \alpha]}\right\|_{p} .
\end{aligned}
$$


Also

$$
\begin{aligned}
\left\|\left(e_{\alpha}-1\right) g^{\prime}\right\|_{p} & =\left(\int_{0}^{\alpha}\left|\frac{t}{\alpha}-1\right|^{p}\left|g^{\prime}(t)\right|^{p} \mathrm{~d} t\right)^{1 / p} \\
& \leq\left\|g^{\prime} \chi_{[0, \alpha]}\right\|_{p} \quad \text { as }\left|\frac{t}{\alpha}-1\right| \leq 1 \quad \text { for } t \in[0, \alpha] .
\end{aligned}
$$

Thus $\left\|\left|e_{\alpha} g-g\left\|\mid \leq\left(p^{-1 / p}+1\right)\right\| g^{\prime} \chi_{[0, \alpha]} \|_{p} \rightarrow 0\right.\right.$ as $\alpha \rightarrow 0+$ because $g^{\prime} \in L^{p}(I), 1 \leq$ $p<\infty$.

Next, if $g \in A C_{\infty}$ and $\left\|g^{\prime}\right\|_{\infty}=K$ then $|g(t)|=\left|\int_{0}^{t} g^{\prime}(s) \mathrm{d} s\right| \leq K t$ and $\left|(t g)^{\prime}\right|=\left|t g^{\prime}+g\right| \leq$ $2 K t$ for all $t \in I$. So

$$
|||t g-t|\|=\| t g^{\prime}+g-1 \|_{\infty} \geq \lim _{t \rightarrow 0}|1-2 K t|=1 .
$$

So $A C_{\infty}$ has no approximate identity. We now find the maximal ideal space $\triangle\left(A C_{p}\right)$ of $A C_{p}$.

Clearly, $A C_{p}$ separates strongly the points of $[0,1]$ and is self-adjoint. If $f \in A C_{p}$ and $a=\inf _{t}|1-f(t)|>0$, take $g=\frac{f}{f-1}$. Then $g^{\prime}=-\frac{f^{\prime}}{(f-1)^{2}}$, so that $\left|g^{\prime}\right| \leq \frac{\left|f^{\prime}\right|}{a^{2}} \in L^{p}(I)$, i.e., $g \in A C_{p}$ and $g \circ f=g+f-g f=0$. Thus $f$ is quasiregular in $A C_{p}$. It follows using Corollary 3.2.8 of Rickart [7] that the maximal ideal space $\triangle\left(A C_{p}\right)$ of $A C_{p}$ is homeomorphic to $\triangle(C(I))=I$ under the natural embedding. Since for $f \in A C_{p}, \hat{f}(0)=f(0)=0$ we get that $\triangle\left(A C_{p}\right)$ of $A C_{p}$ is homeomorphic to $(0,1]$.

Finally, for $r>p, f \in A C_{r}$ we have $f^{\prime} \in L^{r}(I) \subset L^{p}(I)$, so that $f \in A C_{p}$. The inclusion $A C_{r} \subset A C_{p}$ is indeed proper as $L^{r}(I)$ is a proper subset of $L^{p}(I)$ for $r>p$. This completes the proof.

Note that the approximate identity $\left\{e_{\alpha}\right\}$ is bounded if $p=1$ and is unbounded if $1<$ $p<\infty$. Also with our methods the case $p=\infty$ has been solved completely whereas in [1] the maximal ideal space of $L^{\infty}(I)$ could not be calculated.

\section{The multiplier space}

A mapping $T$ on a commutative Banach algebra $A$ to itself is called a multiplier if $T(x y)=$ $x T(y)=T(x) y, x, y \in A$. If $A$ is semisimple and $T: A \rightarrow A$ is a multiplier then there exists a unique bounded continuous function $m$ on $\triangle(A)$ such that $\hat{T} x=m \hat{x}$ for all $x \in A$ and $\|m\|_{\infty} \leq\|T\|$ (p. 19 of [4]). Since for $g \in A C_{p}, \hat{g}(t)=g(t), t \in(0,1], A C_{p}$ is a semisimple Banach algebra, a multiplier $T: A C_{p} \rightarrow A C_{p}$ is a map satisfying $T g=m g, g \in A C_{p}$ for some continuous bounded function $m$ on $(0,1]$. The following theorem gives necessary and sufficient conditions for $m \in C_{b}(0,1]$ to be a multiplier of $A C_{p}$.

Theorem 3. A map $T: A C_{p} \rightarrow A C_{p}, 1 \leq p \leq \infty$ is a multiplier iff there exists an $m \in$ $C_{b}(0,1]$ such that for each $\varepsilon>0, m$ is absolutely continuous on $[\varepsilon, 1], m^{\prime} \in L^{p}[\varepsilon, 1]$ and $\left\|m^{\prime} \chi_{[\varepsilon, 1]}\right\|_{p}=O\left(\varepsilon^{-1 / p^{\prime}}\right)$. (Treat $\varepsilon^{-1 / p^{\prime}}=1$ for $p=1$.)

Proof. Suppose $T$ is a multiplier of $A C_{p}$. Then there exists $m \in C_{b}(0,1]$ such that $T g=m g$. Let the norm of the multiplication operator be $N$. Then $\||T g|\||=||| m g|\|\leq N|\|g \mid\|, g \in$ $A C_{p}$.

As $m e_{\alpha} \in A C_{p}$ and $e_{\alpha}=1$ on $[\alpha, 1]$, we get that $m$ is absolutely continuous on $[\alpha, 1], m^{\prime} \in L^{p}[\alpha, 1]$ and $\left\|m e_{\alpha}\right\||\leq N|\left\|e_{\alpha}\right\|\|=N\| e_{\alpha}^{\prime} \|_{p}=N \alpha^{-1 / p^{\prime}}$. 
Also for $1 \leq p<\infty$,

$$
\begin{aligned}
\left\|\left|e_{\alpha} \|\right|^{p}\right. & =\left\|m^{\prime} e_{\alpha}+m e_{\alpha}^{\prime}\right\|_{p}^{p} \\
& =\int_{0}^{\alpha}\left|m^{\prime} e_{\alpha}+m e_{\alpha}^{\prime}\right|^{p}(t) \mathrm{d} t+\int_{\alpha}^{1}\left|m^{\prime} e_{\alpha}+m e_{\alpha}^{\prime}\right|^{p}(t) \mathrm{d} t \\
& \geq \int_{\alpha}^{1}\left|m^{\prime}\right|^{p}(t) \mathrm{d} t \quad \text { as } e_{\alpha}=1 \text { and } e_{\alpha}^{\prime}=0 \text { on }[\alpha, 1] .
\end{aligned}
$$

So $\left(\int_{\alpha}^{1}\left|m^{\prime}\right|^{p}(t) \mathrm{d} t\right)^{1 / p} \leq N \alpha^{-1 / p^{\prime}}$ or $\left\|m^{\prime} \cdot \chi_{[\alpha, 1]}\right\|_{p}=O\left(\alpha^{-1 / p^{\prime}}\right)$. For $p=\infty$,

$$
\begin{aligned}
\left\|m e_{\alpha}\right\| & =\left\|m^{\prime} e_{\alpha}+m e_{\alpha}^{\prime}\right\|_{\infty} \\
& \geq\left\|\left(m^{\prime} e_{\alpha}+m e_{\alpha}^{\prime}\right) \cdot \chi_{[\alpha, 1]}\right\|_{\infty} \\
& =\left\|m^{\prime} \cdot \chi_{[\alpha, 1]}\right\|_{\infty}
\end{aligned}
$$

and

$$
\left|\left\|m e _ { \alpha } \left|\left\|\leq N\left|\left\|e_{\alpha} \mid\right\|=N\left\|e_{\alpha}^{\prime}\right\|_{\infty}=N / \alpha\right.\right.\right.\right.\right.
$$

so that

$$
\left\|m^{\prime} \cdot \chi_{[\alpha, 1]}\right\|_{\infty}=O(1 / \alpha) .
$$

Conversely, suppose $m \in C_{b}(0,1]$ satisfies, $m$ is absolutely continuous on $[\varepsilon, 1], m^{\prime} \in$ $L^{p}[\varepsilon, 1],\left\|m^{\prime} \chi_{[\varepsilon, 1]}\right\|_{p}=O\left(\varepsilon^{-1 / p^{\prime}}\right)$ for $\varepsilon>0$ and $g \in A C_{p}$. As $g(0)=0$ we have that $m g$ has a continuous extension to $[0,1]$ by assigning $m g(0)=0$. We first show that $(m g)^{\prime} \in L^{p}(I)$. Now $(m g)^{\prime}=m g^{\prime}+m^{\prime} g$. Since $m \in C_{b}(0,1]$ and $g^{\prime} \in L^{p}(I)$, we get that $m g^{\prime} \in L^{p}(I)$. It remains to prove that $m^{\prime} g \in L^{p}(I)$.

For $p=1, p^{\prime}=\infty$ and $\left\|m^{\prime} \chi_{[\varepsilon, 1]}\right\|_{1}=O(1)$ implies that $\lim _{\varepsilon \rightarrow 0} \int_{\varepsilon}^{1}\left|m^{\prime}(t)\right| \mathrm{d} t$ exists, so $m^{\prime} \in L^{1}(I)$. Also $g \in A C_{1}$ is bounded so $m^{\prime} g \in L^{1}(I)$ and $\left\|m^{\prime} g\right\|_{1} \leq\left\|m^{\prime}\right\|_{1}\left\|g^{\prime}\right\|_{1}$, using eq. (1).

For $1<p<\infty$ and $0<\alpha \leq 1 / 4$,

$$
\begin{aligned}
\int_{\alpha}^{2 \alpha}\left|m^{\prime}(s)\right|^{p}|g(s)|^{p} \mathrm{~d} s & \leq \int_{\alpha}^{2 \alpha}\left|m^{\prime}(s)\right|^{p}\left(\int_{0}^{s}\left|g^{\prime}(t)\right| \mathrm{d} t\right)^{p} \mathrm{~d} s \\
& \leq\left(\int_{0}^{2 \alpha}\left|g^{\prime}(t)\right| \mathrm{d} t\right)^{p} \cdot A \cdot \alpha^{-p / p^{\prime}}, \text { where } A \text { is a constant } \\
& =A\left(\int_{0}^{2 \alpha}\left|g^{\prime}(t)\right| \mathrm{d} t\right)^{p} \cdot \alpha^{1-p} \\
& =B\left(\int_{0}^{2 \alpha}\left|g^{\prime}(t)\right| \mathrm{d} t\right)^{p} \int_{2 \alpha}^{4 \alpha} s^{-p} \mathrm{~d} s, \\
& \text { where } B=\frac{A(p-1)}{2^{1-p}-4^{1-p}} \text { is a constant, } \\
& \leq B \int_{2 \alpha}^{4 \alpha} s^{-p}\left(\int_{0}^{s}\left|g^{\prime}(t)\right| \mathrm{d} t\right)^{p} \mathrm{~d} s \\
= & B \int_{2 \alpha}^{4 \alpha}\left(\frac{1}{s} \int_{0}^{s}\left|g^{\prime}(t)\right| \mathrm{d} t\right)^{p} \mathrm{~d} s .
\end{aligned}
$$


Adding these inequalities for $\alpha=1 / 4,1 / 8,1 / 16, \ldots$, we get

$$
\int_{0}^{1 / 2}\left|m^{\prime}(s)\right|^{p}|g(s)|^{p} \mathrm{~d} s \leq B \int_{0}^{1}\left(\frac{1}{s} \int_{0}^{s}\left|g^{\prime}(t)\right| \mathrm{d} t\right)^{p} \mathrm{~d} s \leq B\left(\frac{p}{p-1}\left\|g^{\prime}\right\|_{p}\right)^{p}<\infty
$$

as $\frac{1}{s} \int_{0}^{s}\left|g^{\prime}(t)\right| \mathrm{d} t \in L^{p}(0,1]$ by Hardy's inequality [8]. Also, $\int_{1 / 2}^{1}\left|m^{\prime}(s)\right|^{p}|g(s)|^{p} \mathrm{~d} s \leq$ $\left\|g^{\prime}\right\|_{p}^{p} \cdot A \cdot(1 / 2)^{-p / p^{\prime}}<\infty$, using eq. (1) so that $m^{\prime} g \in L^{p}(I)$ and $\left\|m^{\prime} g\right\|_{p} \leq\left(B\left(\frac{p}{p-1}\right)^{p}+\right.$ $\left.A(1 / 2)^{-p / p^{\prime}}\right)^{1 / p}\left\|g^{\prime}\right\|_{p}$.

For $p=\infty$, let $g \in A C_{\infty}$. Then $|g(t)|=\left|\int_{0}^{t} g^{\prime}(s) \mathrm{d} s\right| \leq t\left\|g^{\prime}\right\|_{\infty}$.

Now $\left|m^{\prime} g \chi_{\left(\frac{1}{2^{n}}, \frac{1}{2^{n-1}}\right]}\right| \leq A 2^{n}\left\|g^{\prime}\right\|_{\infty} \frac{1}{2^{n-1}}=2 A\left\|g^{\prime}\right\|_{\infty}, n=1,2,3, \ldots$

Therefore $\left\|m^{\prime} g\right\|_{\infty} \leq 2 A\left\|g^{\prime}\right\|_{\infty}<\infty$ or $m^{\prime} g \in L^{\infty}(I)$. That $m g$ is absolutely continuous follows from Lemma 1 as $m g$ is absolutely continuous on $[\varepsilon, 1]$ for all $\varepsilon>0$, continuous at 0 and $(m g)^{\prime} \in L^{p}(I) \subseteq L^{1}(I)$. Thus $m$ is a multiplier. It is easy to see that the norm of the multiplier $T$ given by $m$ is

$$
\|T\|=\sup _{\left\|g^{\prime}\right\|_{p}=1}\left\|m^{\prime} g+m g^{\prime}\right\|_{p} \leq\left\{\begin{array}{cl}
\|m\|_{\infty}+\left\|m^{\prime}\right\|_{1}, & p=1 \\
\|m\|_{\infty}+\left(B\left(\frac{p}{p-1}\right)^{p}\right. & \\
\left.+A(1 / 2)^{-p / p^{\prime}}\right)^{1 / p}, & 1<p<\infty \\
\|m\|_{\infty}+2 A, & p=\infty
\end{array} .\right.
$$

This completes the proof.

Remark. For $p=1, m \in C_{b}(0,1]$ is a multiplier of $A C_{1}$ iff $m$ is absolutely continuous on $[\varepsilon, 1]$ for each $\varepsilon>0$ and $m^{\prime} \in L^{1}(I)$, i.e., iff $m$ is absolutely continuous on $I$, using Lemma 1 as $m$ being bounded can be continuously extended to $[0,1]$. Thus Theorem 3 includes the results of [5].

Multipliers from $A C_{r}$ to $A C_{p}, r \neq p$ are given by continuous functions $m \in C(0,1]$ which may not be bounded.

Theorem 4. If $T: A C_{r} \rightarrow A C_{p}$ is a multiplier, $r<p \leq \infty$, then $T=0$.

Proof. Let $T: A C_{r} \rightarrow A C_{p}$ be a multiplier, $r<p \leq \infty$. Then there exists a function $m \in$ $C(0,1]$ such that $T g=m g$. If $m \neq 0$, then there exists $s_{0} \in(0,1]$ such that $m\left(s_{0}\right) \neq 0$. By continuity there is an $\varepsilon>0$ such that $|m(t)|>k / 2$ for $t \in\left(s_{0}-\varepsilon, s_{0}+\varepsilon\right)=N$, say. Here $k=\left|m\left(s_{0}\right)\right|$.

For $t \in N$,

$$
\begin{aligned}
\left|(m g)^{\prime}(t)\right| & =\left|\left(m^{\prime} g+m g^{\prime}\right)(t)\right| \\
& \geq\left|m(t) g^{\prime}(t)\right|-\left|m^{\prime}(t) g(t)\right| \\
& \geq \frac{k}{2}\left|g^{\prime}(t)\right|-\left|m^{\prime}(t) g(t)\right|
\end{aligned}
$$

or

$$
\frac{k}{2}\left|g^{\prime}(t)\right| \leq\left|(m g)^{\prime}(t)\right|+\left|m^{\prime}(t) g(t)\right| .
$$

For $g=e_{\alpha}, \alpha<s_{0}-\varepsilon$ and $t \in N,(m g)^{\prime}(t)=m^{\prime}(t) g(t)+m(t) g^{\prime}(t)=m^{\prime}(t)$. Thus $m^{\prime} \in$ $L^{p}(N)$.

From $(*), \frac{k}{2}\left|g^{\prime}(t)\right| \leq\left|(m g)^{\prime}(t)\right|+\left|m^{\prime}(t)\right|\|g\|_{r} \in L^{p}(N)$ for all $g \in A C_{r}$. A contradiction. So $m=0$. 
The multipliers from $A C_{r}$ to $A C_{p}, r>p$ are given by continuous functions on $(0,1]$ which are locally in $A C_{p}$. The following theorem provides necessary growth conditions on $m^{\prime}$ for $m$ to be a multiplier from $A C_{r}$ to $A C_{p}, r>p$.

Theorem 5. Let $T: A C_{r} \rightarrow A C_{p}, r>p$ be a multiplier given by $T g=m g, g \in A C_{r}$. Then for $\varepsilon>0, m$ is absolutely continuous on $[\varepsilon, 1], m^{\prime} \in L^{p}[\varepsilon, 1]$ and $\left\|m^{\prime} \chi_{[\varepsilon, 1]}\right\|_{p}=O\left(\varepsilon^{-1 / r^{\prime}}\right)$.

Proof. (Similar to the proof of Theorem 3.) Let the norm of the multiplication operator $T$ be $N$. Then $\left\|\left|T g\|\|=\left\||| m g\left|\left\|\leq N\left|\|g \mid\|, g \in A C_{r}\right.\right.\right.\right.\right.\right.$ where the norm on the left-hand side is in $A C_{p}$ and the norm on the right-hand side is in $A C_{r}$.

Since $e_{\alpha} \in A C_{r},\left\|\left(m e_{\alpha}\right)^{\prime}\right\|_{p} \leq N\left\|e_{\alpha}^{\prime}\right\|_{r}$ gives (as in Theorem 3) $\left\|m^{\prime} \chi_{[\alpha, 1]}\right\|_{p} \leq N \alpha^{-1 / r^{\prime}}$ so that $\left\|m^{\prime} \chi_{[\alpha, 1]}\right\|_{p}=O\left(\alpha^{-1 / r^{\prime}}\right)$.

The following theorem gives sufficient conditions to be satisfied by $m \in C(0,1]$ to be a multiplier from $A C_{r}$ to $A C_{p}, r>p>1$.

Theorem 6. If $r>p>1, m \in L^{v}(I) \cap C(0,1]$, where $\frac{1}{v}=\frac{1}{p}-\frac{1}{r}, m$ is absolutely continuous on $[\varepsilon, 1]$ for all $\varepsilon>0$ and $\sum_{n=1}^{\infty}\left(2^{-n / r^{\prime}}\left\|P_{n} m^{\prime}\right\|_{p}\right)^{p}<\infty$, then $T g=m g$ defines a multiplier from $A C_{r}$ to $A C_{p}$. Here $P_{n} m^{\prime}=m^{\prime} \cdot \chi_{\left(2^{-n}, 2^{-n+1}\right]}$.

Proof. For $g \in A C_{r}, g(0)=0$. Since $m \in L^{v}(I) \cap C(0,1], m(t)=O\left(t^{-1 / v}\right)$ in a neighbourhood of 0 so that $|m g(t)| \leq A\left\|g^{\prime}\right\|_{r} t^{-1 / v+1 / r^{\prime}}=A\left\|g^{\prime}\right\|_{r} t^{1 / p^{\prime}}$, using (1). As $p>1, p^{\prime}<\infty$ we get $\lim _{t \rightarrow 0} m g(t)=0$ so $m g$ can be continuously extended to $[0,1]$ by assigning $m g(0)=0$. Now $(m g)^{\prime}=m^{\prime} g+m g^{\prime}$. Since $m \in L^{v}(I)$ and $g^{\prime} \in L^{r}(I)$, it follows that $m g^{\prime} \in L^{p}(I)$. We show that $m^{\prime} g \in L^{p}(I)$. Replacing $g(t)$ by $\int_{0}^{t} g^{\prime}(s) \mathrm{d} s$ we get for $n=1,2,3, \ldots$,

$$
\begin{aligned}
\int_{2^{-n}}^{2^{-n+1}}\left|m^{\prime} g\right|^{p}(t) \mathrm{d} t & \leq \int_{2^{-n}}^{2^{-n+1}}\left|m^{\prime}(t)\right|^{p}\left(\int_{0}^{t}\left|g^{\prime}(s)\right| \mathrm{d} s\right)^{p} \mathrm{~d} t \\
& \leq \int_{2^{-n}}^{2^{-n+1}}\left|m^{\prime}(t)\right|^{p}\left(\left\|g^{\prime}\right\|_{r} t^{1 / r^{\prime}}\right)^{p} \mathrm{~d} t, \quad \text { using (1) } \\
& \leq\left\|g^{\prime}\right\|_{r}^{p}\left(\frac{1}{2^{n-1}}\right)^{p / r^{\prime}} \int_{2^{-n}}^{2^{-n+1}}\left|m^{\prime}(t)\right|^{p} \mathrm{~d} t
\end{aligned}
$$

Adding for $n=1,2,3, \ldots$, we get

$$
\left\|m^{\prime} g\right\|_{p} \leq\left\|g^{\prime}\right\|_{r}\left(\sum_{n=1}^{\infty} 2^{-(n-1) p / r^{\prime}} \int_{2^{-n}}^{2^{-n+1}}\left|m^{\prime}(t)\right|^{p} \mathrm{~d} t\right)^{1 / p}<\infty
$$

since $g^{\prime} \in L^{r}(I)$ and $\sum_{n=1}^{\infty}\left(2^{-n / r^{\prime}}\left\|P_{n} m^{\prime}\right\|_{p}\right)^{p}<\infty$. Thus $(m g)^{\prime} \in L^{p}(I) \subseteq L^{1}(I)$. That $m g$ is absolutely continuous on $I$ follows from Lemma 1 so that $m g \in A C_{p}$. This completes the proof. 
Remarks.

(1) For $f \in L^{r}(I), t^{\frac{1}{r}-\frac{1}{p}-1} \int_{0}^{t} f(s) \mathrm{d} s \in L^{p}(I)$ for $r \leq p$, see [6]. The generalized Hardy's inequality is not available in the case of $r>p$ as in the case of $r=p$, so we get a set of necessary conditions and another set of sufficient conditions for multipliers in the case $r>p$.

(2) It is easy to see that the condition $\left\|m^{\prime} \chi_{[\varepsilon, 1]}\right\|_{p}=O\left(\varepsilon^{-1 / r^{\prime}}\right)$ is equivalent to $\sup _{n} 2^{-n / r^{\prime}}\left\|P_{n} m^{\prime}\right\|_{p}<\infty$.

(3) The results of Theorems 3 and 4 compare with those in [2] in view of Remark 2. In [3], we get that $m$ is a multiplier from $L^{r}(I)$ to $L^{p}(I), r>p$ if $m \in L^{v}(I)$ and $\sum_{n=1}^{\infty}\left(2^{-n / r^{\prime}}\left\|P_{n} m^{\prime}\right\|_{p}\right)^{v}<\infty$, since $\frac{1}{v}=\frac{1}{p}-\frac{1}{r}<\frac{1}{p}, p<v$ and $\ell^{p} \subset \ell^{v}$. Thus the results obtained in Theorem 6 are contained in the results of [3] whereas the necessary conditions in Theorem 5 compare with those in [3].

The following examples have bearing on the above said necessary and sufficient conditions.

Example 1. If $m(t)=t^{-1 / v+\delta}, 0<\delta<1 / v$ then $m(t) \in L^{v}(I) \cap C(0,1], m$ is not bounded and $\sum_{n=1}^{\infty}\left(2^{-n / r^{\prime}}\left\|P_{n} m^{\prime}\right\|_{p}\right)^{p}<\infty$. Thus the sufficiency conditions of Theorem 6 are satisfied so $m$ defines a multiplier from $A C_{r}$ to $A C_{p}, r>p>1$.

Example 2. If $m(t)=t^{-1 / v}$ then $m \notin L^{v}(I)$. Also $\sum_{n=1}^{\infty}\left(2^{-n / r^{\prime}}\left\|P_{n} m^{\prime}\right\|_{p}\right)^{p}=\infty$. So $m$ does not satisfy the sufficiency conditions given by Theorem 6 but $\int_{\varepsilon}^{1}\left|m^{\prime}(t)\right|^{p} \mathrm{~d} t=O\left(\varepsilon^{-p / r^{\prime}}\right)$ so that necessary condition given by Theorem 5 is satisfied. However, if we take $g(x)=$ $\int_{0}^{x} t^{-1 / r}(1-\ln t)^{-2 / r} \mathrm{~d} t, x \in I$ then $g \in A C_{r}$ but $m g \notin A C_{p}$ if $r \leq 2 p$ (one can check that $(m g)^{\prime} \notin L^{p}(I)$.) So $m$ does not define a multiplier from $A C_{r}$ to $A C_{p}, p<r \leq 2 p$.

\section{Acknowledgements}

The author is grateful to Prof. H L Vasudeva for many useful discussions and to Prof. Ajit Iqbal Singh of University of Delhi for useful suggestions.

\section{References}

[1] Baker J W, Pym J S and Vasudeva H L, Totally ordered measure spaces and their $L^{p}$ algebras, Mathematika 29 (1982) 42-54

[2] Bhatnagar S and Vasudeva H L, Spaces of multipliers and their pre-duals for the order multiplication on [0,1], Colloq. Math. 94 (2002) 21-36

[3] Bhatnagar S, Spaces of multipliers and their pre-duals for the order multiplication on [0,1] - II, Colloq. Math. 99 (2004) 267-273

[4] Larsen R, An introduction to the theory of multipliers (Berlin, New York: SpringerVerlag) (1971)

[5] Larsen R, The multipliers of $L^{1}[0,1]$ with order convolution, Publ. Math. Debrecen 23 (1976) 239-248

[6] Okikiolu G O, Bounded linear transformations in $L^{p}$ space, J. London Math. Soc. 41 (1966) 407-414

[7] Rickart C E, General theory of Banach algebras (New York: D. Van Nostrand Co.) (1960)

[8] Stein E M and Weiss G, Introduction to Fourier analysis on Euclidean spaces (New Jersey: Princeton) (1971) 